\title{
Design and Performance of Microstrip Diplexers: A Review
}

\author{
Salah I. Yahya ${ }^{1,2}$, Abbas Rezaei $^{3}$ and Leila Nouri ${ }^{4,5}$ \\ ${ }^{1}$ Department of Software Engineering, Faculty of Engineering, Koya University, \\ Koya KOY45, Kurdistan Region - F.R. Iraq \\ ${ }^{2}$ Department of Communication and Computer Engineering, Cihan University-Erbil, \\ Kurdistan Region - F.R. Iraq \\ ${ }^{3}$ Department of Electrical Engineering, Kermanshah University of Technology, \\ Kermanshah, Iran \\ ${ }^{4}$ Institute of Research and Development, Duy Tan University, \\ Da Nang, 550000, Vietnam \\ ${ }^{5}$ Department of Electrical and Electronic Engineering, Duy Tan University, \\ Da Nang 550000, Vietnam
}

\begin{abstract}
The radiofrequency microstrip diplexers are widely demanded nowadays by modern wireless communication systems. Hence, several types of previously reported microstrip diplexers are reviewed and investigated in this work. Microstrip diplexers are three ports devices used for separating desired signals and delivering them through two (or more) different channels. The diplexers are investigated in three categories of dual-channel bandpass-bandpass diplexers, multichannel diplexers, and lowpassbandpass diplexers. The investigated multi-channel diplexers include a number of four-channel, six-channel, and eight-channel diplexers. Due to the hard design process, the number of reported diplexers with more than four channels is limited. The layout structures and theory design methods of the previously reported diplexers are studied. Moreover, their size and performance are compared while some explanations about their advantages and disadvantages are presented. This comparison includes insertion loss, return loss, fractional bandwidths, isolation, selectivity, and gaps between channels.
\end{abstract}

Index Terms-Microstrip, Diplexer, Bandpass-bandpass, Lowpass-bandpass, Multichannel.

\section{INTRODUCTION}

High-performance and compact microstrip devices are strongly demanded by modern telecommunication and wireless networks. These microstrip devices are microstrip filters, couplers, diplexers, multiplexers, power dividers,

ARO-The Scientific Journal of Koya University Vol. VIII, No.1 (2020), Article ID: ARO.10634, 12 pages DOI:10.14500/aro.10634

Received 12 January 2019; Accepted 12 March 2020

Review paper: Published 15 March 2020

Corresponding author's e-mail: leilanouri@duytan.edu.vn Copyright (C) 2020 Salah I. Yahya, Abbas Rezaei, Leila Nouri. This is an open-access article distributed under the Creative Commons Attribution License. and sometimes low-noise amplifiers (Noori and Rezaei, 2017; Shen and Che, 2020; Salehi and Noori, 2014; Chen, et al., 2019; Rezaei and Noori, 2018; Salehi, et al., 2016). A microstrip diplexer is a passive three-port device for frequency-domain multiplexing. It usually consists of two filters which are integrated by a junction circuit (Feng, et al., 2017; Huang, et al., 2016). The signal can be transmitted to the other two ports through a common port connected to the junction circuit (Rezaei, et al., 2019). Each diplexer has two channels working at two frequencies for the specific applications. For examples, the proposed diplexer in Yang, et al., 2014, has been designed for 4G application while the introduced diplexer in Noori and Rezaei, 2017, has been designed for IEEE 802.16 and 802.20 WiMAX technology and wireless applications. When the channels are close together, the diplexer can be employed for frequency division duplex (FDD) scheme (Peng and Chiang, 2015). However, in this case, improving the insertion loss and isolation between channels is hard (Noori and Rezaei, 2017). The diplexers can be formed by two bandpass filters (BPF), which are bandpass-bandpass diplexers (BBDs) (Jun-Mei, et al., 2016; Chinig, et al., 2015; Guan, et al., 2014; Chen, et al., 2015; Xiao, et al., 2015; Rezaei and Noori, 2018; Sasipriya and Aparna, 2018; Rezaei and Noori, 2018). Some of BBDs have two channels (Deng, et al., 2013; Noori and Rezaei, 2017; Rezaei, et al., 2017; Feng, et al., 2014; Chinig, et al., 2015; Salehi, et al., 2016; Chen, et al., 2006; Cheng, et al., 2013; Chinig, 2017; Wang, et al., 2016) named dual-channel diplexers. However, some have more than two channels. The multiplexers have more than three ports and more than two channels (Heng, et al., 2014), but multi-channel diplexers have only three ports with the channel numbers more than two (Liu, et al., 2017; Lee, et al., 2016; Wu, et al., 2013; Lai and Jeng, 2005; Hsu, et al., 2016). Since ultra-wideband has traditional applications in non-cooperative radar, some of diplexers have been designed with wide channels 
(Deng, et al., 2013). The wideband diplexer can use a very low energy level for short-range and high-bandwidth communications. On the other hand, a number of diplexers have narrow channels (Salehi, et al., 2016). They are suitable for multi-channel long-range RF communication systems (Noori and Rezaei, 2018; Yahya, et al., 2019). The stopband rejection of a high-performance diplexer must be wide with low harmonic level (Yahya, et al., 2019; Bukuru, et al., 2015; Rezaei, et al., 2019). Nevertheless, many designers did not give attention to attenuate the harmonics (Rezaei, et al., 2019; Rezaei, et al., 2019). Other types of diplexers are lowpass-bandpass diplexers (LBDs), which have been formed by a lowpass filter (LPF), BPF, and junction circuit (Rezaei, et al., 2019; Deng and Tsai, 2013; Rayatzadeh and Moloudian, 2019; Heshmati and Roshani, 2018; Capstick, 1999; Hayati, et al., 2019). An important factor related to the diplexer performance is the high selectivity. However, some of the diplexer designers did not improve the selectivity (Rezaei, et al., 2019; Bui, et al., 2017) while the others could design the diplexers with high-frequency selectivity at both channels (Rezaei and Noori, 2018; Lobato-Morales, et al., 2012). A well-designed diplexer must have low loss and high isolation between channels. For example, a microstrip diplexer that reported in Ghafari and Afsahi, 2019, has relatively a good isolation but large measured insertion and return losses at both channels. Furthermore, a diplexer with flat passbands has low time distortion, which is an advantage.

In this work, several kinds of microstrip diplexers are reviewed. The structures and performance of these diplexers are studied to find the best structures with high performance and small size. Moreover, the mathematical methods of analyzing some structures have been reviewed. The Frequency response and overall size of the introduced diplexers are compared in the following categories: Dualchannel BBDs, multichannel bandpass diplexers, and LBDs.

\section{DUAL-CHANNEL BBDs}

The majority designed diplexers for the wireless communication market are the dual-channel BBDs. Usually, to design this type of diplexer, the designers use two similar BPF but with different overall dimensions. Each filter should create only one passband. Therefore, to design a dual-channel diplexer, we need two single-mode resonators. Several structures have been proposed to design the dual-band bandpass diplexers (Feng, et al., 2017; Huang, et al., 2016; Rezaei, et al., 2019; Yang, et al., 2014; Noori and Rezaei, 2017; Peng and Chiang, 2015; Jun-Mei, et al., 2016; Chinig, et al., 2015; Guan, et al., 2014; Chen, et al., 2015; Xiao, et al., 2015; Rezaei and Noori, 2018; Sasipriya and Aparna, 2018; Rezaei and Noori, 2018; Deng, et al., 2013; Noori and Rezaei, 2017; Rezaei, et al., 2017; Feng, et al., 2014; Chinig, et al., 2015; Salehi, et al., 2016; Chen, et al., 2006; Cheng, et al., 2013; Chinig, 2017; Wang, et al., 2016). To achieve a high performance, different mathematical methods have been used. Some of the layout configurations of diplexers with a summary of their design methods are presented in Table I. In addition to the presented layout, some lumped elements have been used (Feng, et al., 2017). Due to the symmetric structures, the even/odd modes analysis has been performed (Feng, et al., 2017; Huang, et al., 2016; Yang, et al., 2014; Guan, et al., 2014; Rezaei and Noori, 2018; Sasipriya and Aparna, 2018; Rezaei and Noori, 2018). The even/odd input admittances $\left(Y_{\text {ine }}\right.$ and $\left.Y_{\text {ino }}\right)$ have been calculated to obtain the coupling coefficient $k_{12}$ according to the following formula (Feng, et al., 2017):

$$
k_{12}=\frac{\operatorname{Im}\left[Y_{12}\left(\omega_{o}\right)\right]}{\frac{\omega_{o}}{2} \frac{\partial \operatorname{Im}\left[Y_{11}\left(\omega_{o}\right)\right]}{\partial \omega}} ; Y_{11}=\frac{Y_{\text {ine }}+Y_{\text {ino }}}{2}, Y_{12}=\frac{Y_{\text {ine }}-Y_{\text {ino }}}{2}
$$

Where $\omega_{0}$ is an angular resonance frequency. As mentioned in Feng, et al., 2017, the coupling factor is strongly affected by the gap between resonators. Accordingly, another way to realize $k_{12}$ is by selecting the gap between the resonators and coupling sections. For analyzing the symmetrical resonators (Huang, et al., 2016; Yang, et al., 2014; Guan, et al., 2014; Liu, et al., 2017), the even and odd modes resonance frequencies $f_{\text {even }}$ and $f_{\text {odd }}$ are expressed as:

$$
\begin{aligned}
& f_{\text {odd }}=\frac{(2 n-1) c}{2 L_{1} \sqrt{\varepsilon_{\text {eff }}}} \text { and } f_{\text {even }}=\frac{n c}{\left(L_{1}+2 L_{2}\right) \sqrt{\varepsilon_{\text {eff }}}} \\
& \text { for }: n=1,2,3, \ldots
\end{aligned}
$$

Where $\varepsilon_{\text {eff }}$ is the effective dielectric constant and $c$ is the speed of light in free space. The physical length of half circuit under odd-mode and even mode excitations are depicted by $L_{1}$ and $L_{2}$, respectively. As depicted in Table II, the resonators (Huang, et al., 2016; Yang, et al., 2014) are similar where they are loaded by similar T-shape stubs. On the other hand, they used (2) to calculate the even/odd mode resonance frequencies. However, they have different junction circuits.

The meandrous lines have been coupled to realize a microstrip diplexer (Rezaei, et al., 2019; Chinig, 2017; Bukuru, et al., 2015), but they are implemented on different substrates. To analyze some resonators, the ABCD matrixes have been calculated (Salehi and Noori, 2014; Noori and Abiri, 2016; Rezaei, et al., 2019; Noori and Rezaei, 2017; Noori and Rezaei, 2017). Using the ABCD matrix (Rezaei, et al., 2019), $S_{21}$ and $Z$ matrix have been calculated. Then for having the lowest insertion loss, the value of $S_{21}$ is obtained. On the other hand, based on the value of $Z_{21}$, the resonance condition is obtained. Finally, by combining the equations, the values of the physical lengths at a target resonance frequency are obtained. Meanwhile, Noori and Rezaei (2017) calculated the reflection coefficient based on the ABCD matrix to obtain the condition of good isolation. However, due to its special structure, it could not improve the isolation between channels. The transmission matrix (Noori and Rezaei, 2017) is utilized based on an equivalent $L C$ circuit of a basic resonator. It is utilized for calculating the values of the unknown stubs dimensions. As shown in Table I, the simple structure (Jun-Mei, et al., 2016) is analyzed by calculating the external quality factors corresponding to source and load $\left(Q_{\mathrm{e}, \mathrm{o}}\right.$ and $\left.Q_{\mathrm{e}, \mathrm{N}+1}\right)$ using the coupling coefficients $\left(M_{0,1}\right.$ and $\left.M_{\mathrm{N}, \mathrm{N}+1}\right)$ as follows: 
TABLE I

Layout Configuration, Substrate, and Theory Method of the Dual-channel BBDs. $\varepsilon_{\mathrm{R}}$ Is the Substrate Relative Dielectric Constant and $H$ is the Substrate Height

\begin{tabular}{|c|c|c|c|}
\hline Refs. & Diplexer structure & Substrate & Theory method \\
\hline (Feng, et al., 2017) & & $\begin{array}{c}\varepsilon_{\mathrm{r}}=2.65 \\
h=0.508 \mathrm{~mm}\end{array}$ & $\begin{array}{l}\text { 1.Calculating the even/odd modes admittances }\left(Y_{\text {ine }} \text { and } Y_{\text {ino }}\right) \\
\text { 2.Calculating the coupling coefficient }\end{array}$ \\
\hline
\end{tabular}

(Huang, et al., 2016)

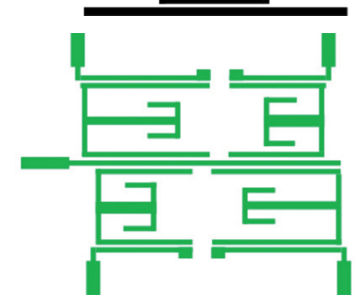

(Rezaei, et al., 2019)

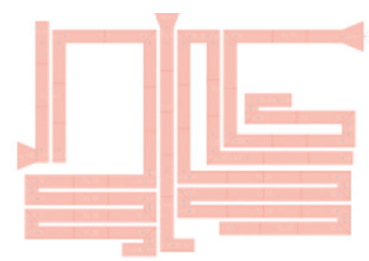

(Yang, et al., 2014)

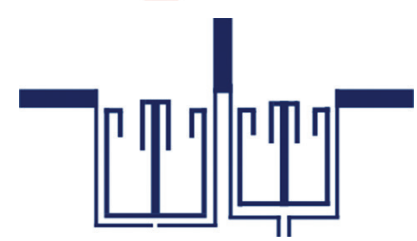

(Noori and Rezaei, 2017)

(Jun-Mei, et al., 2016)

(Chinig, et al., 2015)
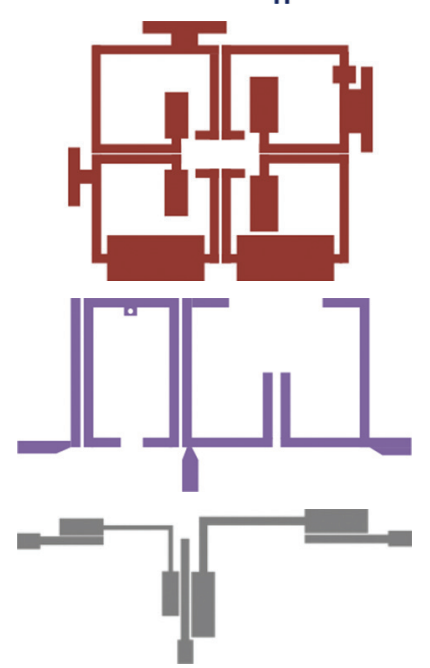

(Guan, et al., 2014)

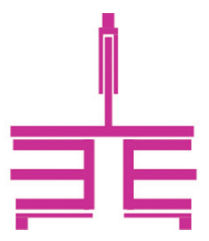

(Chen, et al., 2015)

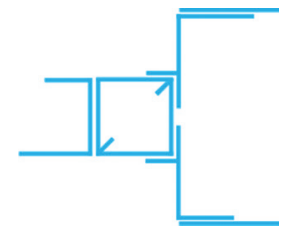

$\varepsilon_{\mathrm{r}}=2.55$

$h=0.8 \mathrm{~mm}$

$\varepsilon_{\mathrm{r}}=4.4$

$h=1.58 \mathrm{~mm}$

Rogers RO4003C Finding the fundamental odd and even modes resonant frequencies $\varepsilon_{\mathrm{r}}=3.55$ $h=0.508 \mathrm{~mm}$

RT_Duroid_5880 1. Calculating the ABCD matrix

$\varepsilon_{\mathrm{r}}=2.2 \quad$ 2. Calculating $\mathrm{S}_{21}$ and $\mathrm{Z}$ matrix from $\mathrm{ABCD}$ matrix

$h=0.787 \mathrm{~mm} \quad 3$. Calculating the resonance frequency from $\mathrm{Z}$ matrix

$\varepsilon_{\mathrm{r}}=3.5$ $h=0.8 \mathrm{~mm}$

Finding the fundamental odd and even modes resonant frequencies

RT_Duroid_5880 1. Calculating the transmission matrix

$\varepsilon_{\mathrm{r}}=2.2 \quad$ 2. Calculating the reflection coefficient

$h=0.787 \mathrm{~mm} \quad 3$. Finding a method to obtain better isolation between two channels

1.Calculating the external quality factors

2.Tuning the space between coupled lines based on quality factors

1. Even and odd modes analysis by calculating the input admittance 2. Calculating the desired frequency ratio of harmonic from the input admittance

$\varepsilon_{\mathrm{r}}=3.5$ $h=0.8 \mathrm{~mm}$

Calculating the even and odd modes of resonance frequencies

$\varepsilon_{\mathrm{r}}=10.8$

$h=0.653 \mathrm{~mm}$
Finding the resonance condition based on the equivalent circuit of quarter resonator 
TABLE I

(CONTINUED)

\begin{tabular}{|c|c|c|c|}
\hline Refs. & Diplexer structure & Substrate & Theory method \\
\hline (Chen, et al., 2015) & & $\begin{array}{c}\text { RT_Duroid_5880 } \\
\varepsilon_{\mathrm{r}}=2.2 \\
h=0.787 \mathrm{~mm}\end{array}$ & $\begin{array}{l}\text { 1. Proposing an equivalent } L c \text { model of the resonator } \\
\text { 2. Even and odd mode analysis using } L c \text { circuit } L c\end{array}$ \\
\hline (Sasipriya and Aparna, 2018) & & $\begin{array}{c}\text { RT_Duroid_5880 } \\
\varepsilon_{\mathrm{r}}=2.2 \\
h=0.787 \mathrm{~mm}\end{array}$ & $\begin{array}{l}\text { 1. Proposing an equivalent } L c \text { model of the resonator } \\
\text { 2. Even and odd mode analysis using } L c \text { circuit }\end{array}$ \\
\hline
\end{tabular}

BBDS: Bandpass-bandpass diplexers

TABLE II

Performance Comparison among Different Reported BBD

\begin{tabular}{|c|c|c|c|c|c|c|c|}
\hline Refs. & IL1, IL2 (dB) & RL1, RL2 (dB) & $\operatorname{Size}\left(\lambda_{\mathrm{g}}^{2}\right)$ & Isolation $(\mathrm{dB})$ & $f_{\mathrm{o} 1}, f_{\mathrm{o} 2}(\mathrm{GHz})$ & $\bar{f} f_{\mathrm{o} 2} / f_{\mathrm{o} 1}(\mathrm{GHz})$ & FBW1, FBW2 (\%) \\
\hline (Feng, et al., 2017) & $1.4,3.4$ & 15,20 & 0.089 & 45 & $1.05,1.87$ & 1.78 & $6.1,4$ \\
\hline (Huang, et al., 2016) & $1,0.9$ & Better than 20 & 0.127 & 30 & $2.3,2.72$ & 1.18 & $6.1,5.8$ \\
\hline (Rezaei, et al., 2019) & $0.36,0.44$ & Better than 23.7 & 0.028 & 23 & $2.88,3.29$ & 1.14 & --- \\
\hline (Noori and Rezaei, 2017) & $0.6,0.9$ & $11.3,12.4$ & $0.076^{*}$ & 13.8 & $2.6,6$ & 2.30 & --- \\
\hline (Peng and Chiang, 2015) & $2.1,2.1$ & Better than 20 & 0.07 & 20 & $1.75,1.85$ & 1.06 & 5,5 \\
\hline (Jun-Mei, et al., 2016) & $2.2,2.1$ & $11.9,12$ & 0.064 & 30 & $1.82,2.41$ & 1.32 & $2.8,1.9$ \\
\hline (Guan, et al., 2014) & $1.2,1.5$ & --- & 0.137 & 35 & $1.95,2.14$ & 1.10 & $3.59,3.27$ \\
\hline (Chen, et al., 2015) & $1.83,1.52$ & --- & 0.705 & 26 & $1.1,1.3$ & 1.18 & $8,9.2$ \\
\hline (Xiao, et al., 2015) & $1.43,1.59$ & --- & 0.282 & 42 & $2.44,3.52$ & 1.44 & --- \\
\hline (Rezaei and Noori, 2018) & $0.14,0.16$ & $18.5,20$ & 0.022 & 34 & $1.8,2.4$ & 1.33 & $11,7.1$ \\
\hline (Rezaei and Noori, 2018) & $0.28,0.29$ & $21.2,24.3$ & 0.010 & 30 & $0.8,0.9$ & 1.12 & $3.2,3.2$ \\
\hline (Deng, et al., 2013) & 3,3 & Better than 10.3 & 0.073 & 37.5 & $3,5.8$ & 1.93 & 80,5 \\
\hline (Salehi, et al., 2016) & $1.5,1.3$ & Better than 21 & $0.087 *$ & 30 & $2.3,2.55$ & 1.10 & $3.6,3.4$ \\
\hline (Chen, et al., 2006) & $2.8,3.2$ & 16,17 & --- & 30 & $1.5,1.75^{*}$ & 1.16 & $3.8,3.3$ \\
\hline (Cheng, et al., 2013) & $2.86,3.04$ & Better than 20 & 0.688 & 40 & 8,9 & 1.12 & --- \\
\hline (Chinig, 2017) & $2.6,2.4$ & 35,33 & $0.079 *$ & 24.4 & $1.7,2.49$ & 1.46 & $2.6^{*}, 5.1^{*}$ \\
\hline (Wang, et al., 2016) & $0.4,0.3$ & Better than 13 & 0.488 & 70 & $9.9,10.02$ & 1.01 & $0.65^{*}, 0.65^{*}$ \\
\hline (Yahya, et al., 2019) & $0.85,0.8$ & $15.7,24$ & 0.047 & 21 & $2.85,2.72$ & 1.05 & --- \\
\hline (Bukuru, et al., 2015) & $1.35,1.31$ & 15,20 & 0.05 & 25 & $3.65,5.2$ & 1.42 & $8.2,7.69$ \\
\hline (Rezaei, et al., 2019) & $0.25,0.26$ & $18.45,17.47$ & 0.038 & 24 & $2.12,3.94$ & 1.85 & --- \\
\hline (Rezaei, et al., 2019) & $0.10,0.16$ & 33,22 & 0.054 & 22 & $1.6,2.1$ & 1.31 & $16.8,11$ \\
\hline (Bui, et al., 2017) & $0.4,0.42$ & better than 20 & 0.095 & --- & $1.8,2.45$ & 2.36 & --- \\
\hline (Rezaei and Noori, 2018) & $0.21,0.21$ & 32,25 & 0.018 & 40 & $1,1.3$ & 1.3 & $4.6,4.6$ \\
\hline
\end{tabular}

*: Approximated values. BBDS: Bandpass-bandpass diplexers

$$
\begin{aligned}
& Q_{e, o}=\frac{1}{F B W M_{0,1}^{2}} \\
& Q_{e, N+1}=\frac{1}{F B W M_{N, N+1}^{2}}
\end{aligned}
$$

Where $M_{\mathrm{i}, \mathrm{i}+1}$ is the coupling coefficient and fractional bandwidth (FBW) is the FBW. Finally, the space between coupled lines is tuned based on quality factors. The resonator analysis presented by Guan, et al. (2014) and Rezaei, et al. (2017) has been performed by calculating the extra quality factor $\left(Q_{\mathrm{e}}\right)$ as defined by the following equation:

$$
Q_{e}=\frac{2 f_{o}}{(\Delta f)_{3 d B} 10^{\frac{-I L}{20}}}
$$

Where $f_{o}$, IL, and $(\Delta f)_{3 \mathrm{~dB}}$ are resonance frequency in $\mathrm{GHz}$, insertion loss in $\mathrm{dB}$, and $-3 \mathrm{~dB}$ bandwidth, respectively. Proposing an approximated $L C$ circuit is a method to analyze the resonator structure. Fig. 1 presents some resonators with their approximated equivalent $L C$ circuit proposed in Noori and Rezaei, 2017; Rezaei and Noori, 2018; Rezaei and Noori, 2018; Salehi, et al., 2016; Rezaei, et al., 2019. Since the effects of steps in widths and bent are negligible 


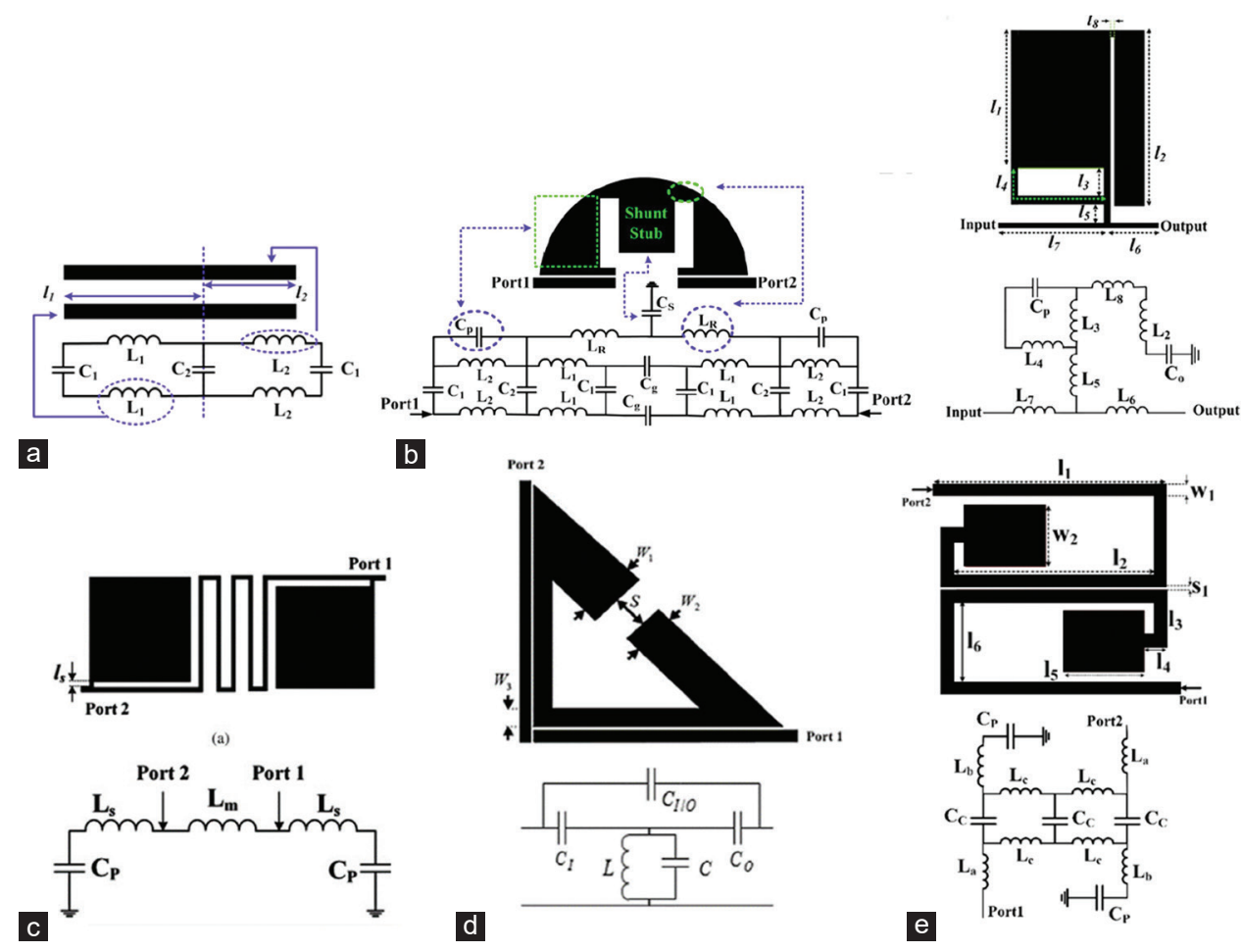

Fig. 1. Layout of some resonators and their equivalent $L C$ circuits reported by: (a) Noori and Rezaei (2017), (b) Rezaei and Noori (2018), (c) Rezaei and Noori (2018), (d) Salehi, et al. (2016), (e) Rezaei, et al. (2019).

at the frequencies lower than $10 \mathrm{GHz}$, the equivalent lumped elements of these sections are removed. The patch cells are replaced by capacitors, and thin stubs are presented by inductors. As depicted in Fig. 1, the effect of coupling between microstrip lines is replaced by some capacitors. Using these $L C$ circuits, it can be easy to calculate the resonance frequencies, $\mathrm{ABCD}$ matrix, input impedance/ admittance, and even/odd modes analysis.

A well-designed diplexer must be compact with low loss, sharp roll-off, attenuated harmonics, and high isolation. The simulated and measured frequency responses of dualchannel BBDs in (Huang, et al., 2016; Jun-Mei, et al., 2016; Guan, et al., 2014; Cheng, et al., 2013) are presented in Figs. 2(a)-(d), respectively. As presented in Fig. 2, the parameters $S_{21}$ and $S_{31}$ show the transition between common port (1) and other ports, while the isolation between channels is depicted by $S_{23}$. However, they could not improve the selectivity while the harmonics did not suppress.

The size and performance of designed dual-band BBDs are compared in Table II. In this table; RL is the return loss, and $f_{\mathrm{o} 1}$ and $f_{\mathrm{ol}}$ are the resonance frequencies of the first and second channels, respectively. As presented in Table II, the lowest insertion losses at both channels are achieved in Rezaei and Noori, 2018, while the best return losses are obtained in Chinig, et al., 2015. The overall sizes of the reported diplexers are presented in $\lambda_{\mathrm{g}}{ }^{2}$, where $\lambda_{\mathrm{g}}$ is the guided wavelength calculated at the lower resonance frequencies. The comparison results show that the most compact dualband BBD is designed in Rezaei and Noori, 2018, with the overall size of $0.01 \lambda_{\mathrm{g}}{ }^{2}$. Another important factor in the diplexer design is the isolation between channels where the highest isolation $(70 \mathrm{~dB})$ is achieved in Wang, et al., 2016. When the channels are close together, the diplexer can be used for FDD applications. Nevertheless, having a small gap between channels leads to increase in the loss and decrease the isolation. Accordingly, the diplexer with $f_{\mathrm{o} 2} / f_{\mathrm{o} 1} \leq 1.1$ has been less designed. As shown in Table II, only the proposed diplexer in Wang, et al., 2016, could reach $f_{\mathrm{o} 2} / f_{\mathrm{o} 1}=1.01$, but it could not improve the common port return loss at both channels. The narrowband and wideband diplexers can be identified from the presented FBWs in Table II. A diplexer with FBW1 $=2.8 \%$ and FBW2 $=1.9 \%$ is designed in JunMei, et al., 2016, where it can be used for long-range RF communication systems. However, having narrow channels increases the group delay that leads to time distortion. On the other hand, a diplexer with $80 \%$ first channel FBW is designed in Deng, et al., 2013, which is suitable for ultrawideband applications.

\section{Multichannel BBDs}

Another type of diplexer is multichannel BBDs. Quadchannel diplexers are a type of BBDs which is the most reported one. The quad-channel diplexers have three ports similar to dual-band BBDs, but with four channels. They are suitable for multichannel communication systems. The special applications of dual and multichannel diplexers are related to their resonance frequencies. For example, a diplexer with resonance frequencies at $2.4 \mathrm{GHz}$ and $5.2 \mathrm{GHz}$ is appropriate for wireless local area networks (WLAN) while a diplexer with an operational frequency at $1.8 \mathrm{GHz}$ is suitable for GSM applications (Rezaei and Noori, 2018). The 

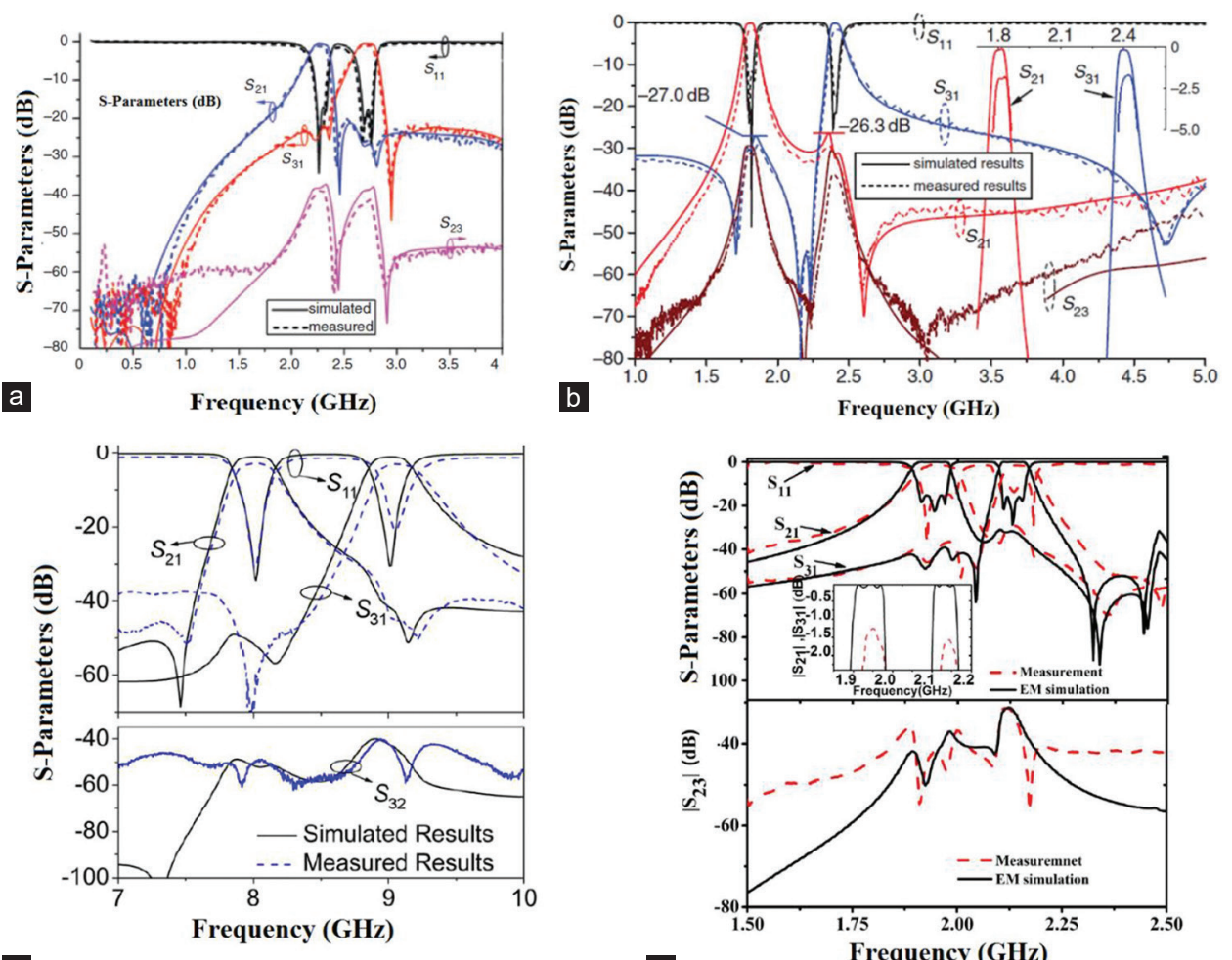

C

Frequency $(\mathbf{G H z})$

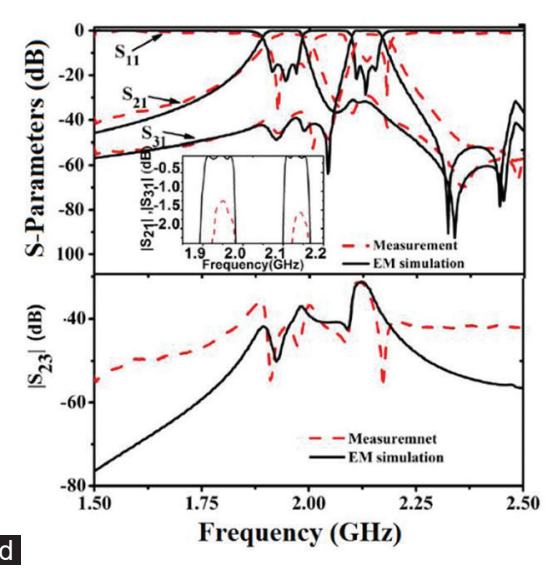

Fig. 2. Simulated and measured frequency responses of dual-channel bandpass-bandpass diplexers, (a) (Huang, et al., 2016), (b) (Jun-Mei, et al., 2016), (c) (Guan, et al., 2014), (d) (Cheng, et al., 2013).

quad-band diplexers are usually composed of two dual-band BPF and a junction circuit. The sizes of this type of diplexer are usually larger than dual-band BBDs. The layout structure, substrate properties, and theory method of the quad-band bandpass diplexers are summarized in Table III.

As illustrated in Table III, the microstrip spiral cells with inductance features have been utilized to design quad-band bandpass diplexers (Heng, et al., 2014; Liu, et al., 2017). They are integrated by murderous microstrip lines. The spiral cells have the advantage of being compact, but the junction circuits (Heng, et al., 2014; Liu, et al., 2017) occupy large implementation areas. When we decrease the gap between spiral cells, they will be coupled to each other. The coupling between them creates some small coupling capacitors. Therefore, the inductor and capacitors can create passbands easily. The quad-band diplexer reported by Heng, et al. (2014) is designed based on proposing an $L C$ circuit and finding the coupling coefficients. However, in Liu, et al., 2017, the even and odd modes resonance frequencies have been calculated as functions of $\varepsilon_{\text {eff }}$ and light speed $c$. This diplexer is designed using BPF. Then, the coupling coefficient from the simulated $S$-parameters is extracted by:

$$
M=\left(f_{o 2}{ }^{2}-f_{o 1}{ }^{2}\right) /\left(f_{o 2}{ }^{2}+f_{o 1}{ }^{2}\right)
$$

Where $f_{\mathrm{o} 1}$ and $f_{\mathrm{o} 2}$ represent the lower and higher resonance frequencies of the proposed filter. To design a microstrip four-channel diplexer in Lee, et al., 2016, the step impedance cells have been used. In this work, the design method is based on the calculation of the resonance frequencies of step
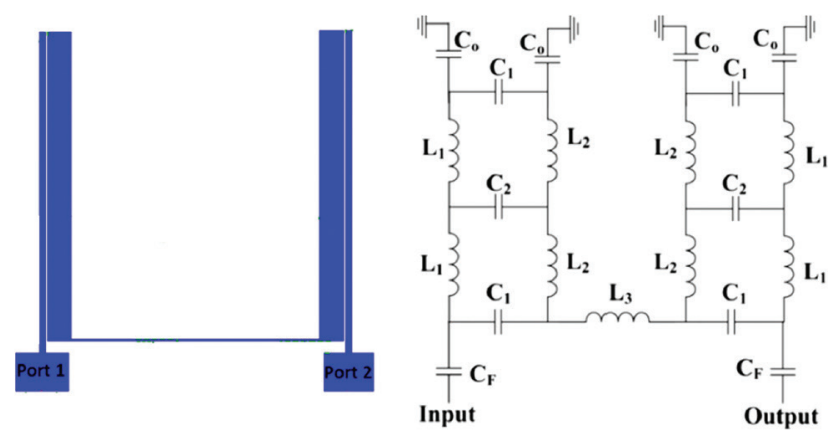

Fig. 3. The basic resonator and its approximated $L C$ circuit presented by Noori and Rezaei (2018).

impedance sections without mathematical formulas. Coupled open loops have been integrated on RT/Duroid 5880 substrate with $\varepsilon_{\mathrm{r}}=2.2$ and $h=0.787 \mathrm{~mm}$ to realize a four-channel diplexer in $\mathrm{Wu}$, et al., 2013. In the loop structures, low impedance sections are utilized. The analysis of this structure is performed based on finding the impedance ratio of the low impedance section. Furthermore, similar to the proposed diplexer in Lee, et al., 2016, the coupling coefficients are obtained from the two resonant modes based on (5) using the full-wave electromagnetic simulator.

Coupled hairpins have been integrated in Hsu, et al., 2016, to obtain a microstrip diplexer with four passbands. This diplexer is designed based on finding the coupling coefficient as functions of the distance between resonators without mathematical formulas. To obtain a four channel diplexer in Noori and Rezaei, 2018, two similar dual-band BPF with 
TABLE III

Layout Configuration, Substrate Type, and Theory Method of the Quad-BBD

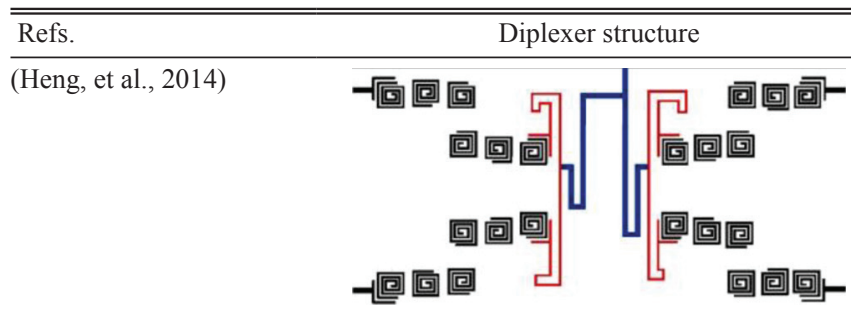

(Liu, et al., 2017)

(Lee, et al., 2016)

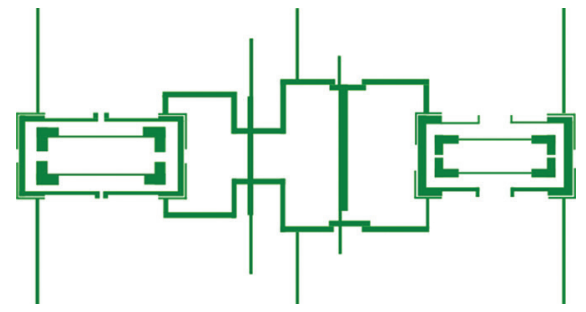

(Wu, et al., 2013)

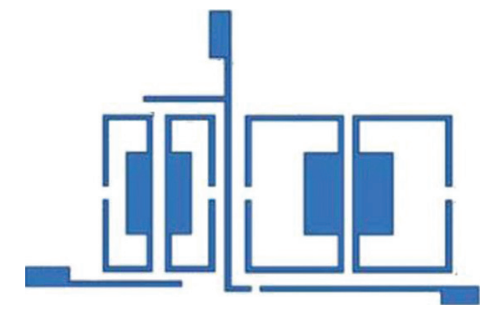

(Hsu, et al., 2016)

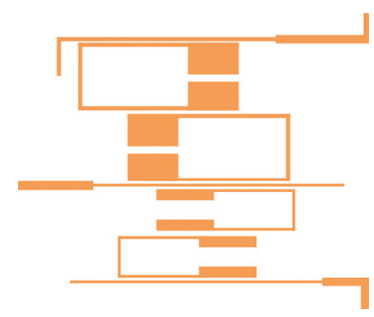

(Noori and Rezaei, 2018)

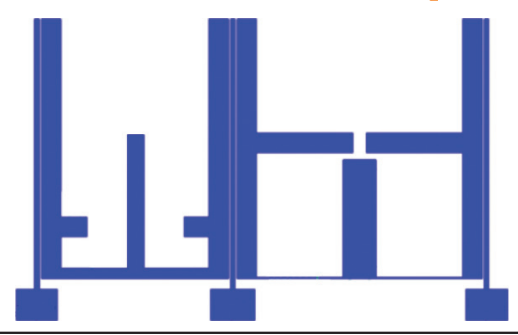

Substrate Theory method

$\mathrm{MgO} \quad$ Proposing an equivalent circuit model for the diplexer

$\varepsilon_{\mathrm{r}}=9.8$

$h=0.5 \mathrm{~mm}$

$\mathrm{MgO}$ 1. Calculating of the external quality factors and

$\varepsilon_{\mathrm{r}}=9.78 \quad$ coupling coefficients of the diplexer

$h=0.5 \mathrm{~mm} \quad 2$. Extracting the coupling coefficients from the simulated $S$-parameters

RT/Duroid 6010

$\varepsilon_{\mathrm{r}}=10.2$

$h=0.635 \mathrm{~mm}$

Calculating the resonance frequencies of the step impedance sections without mathematical formulas

RT/Duroid 5880

$$
\varepsilon_{\mathrm{r}}=2.2
$$

$h=0.787 \mathrm{~mm}$

Analysis based on finding the impedance ratio of the low impedance section
RT/Duroid 6010LM $\varepsilon_{\mathrm{r}}=10.2$ $h=0.635 \mathrm{~mm}$
Calculating the coupling coefficient as functions of the distance between resonators without mathematical formulas
RT/Duroid 5880

$$
\varepsilon_{\mathrm{r}}=2.2
$$

$h=0.787 \mathrm{~mm}$
1. Proposing an $L c$ model for the basic resonator

2. Calculating the angular resonance frequency from $L c$ circuit

BBDS: Bandpass-bandpass diplexers

different dimensions have been designed and analyzed. For this purpose, first, an approximated $L C$ model of the basic resonator is presented. Then, the input impedance of the $L C$ circuit is extracted. Finally, an angular resonance frequency is calculated when the input impedance is zero. In this case, the dimensions can be tuned based on a target resonance frequency when the equations show the resonator behavior. The basic resonator and its approximated $L C$ circuit (Noori and Rezaei, 2018) are presented in Fig. 3. As shown in
Fig. 3, the open ends and feed lines are replaced by the capacitors $C_{\mathrm{o}}$ and $C_{\mathrm{F}}$, respectively.

Similar to the dual-band BBDs, a high-performance quad-band bandpass diplexer must have low loss, highfrequency selectivity, suppressed harmonics, high isolation, and low group delay. The frequency response of the quadband bandpass diplexers (Liu, et al., 2017; Lee, et al., 2016; Hsu, et al., 2016; Noori and Rezaei, 2018) is presented in Figs. 4(a)-(d), respectively. Advanced design system full- 

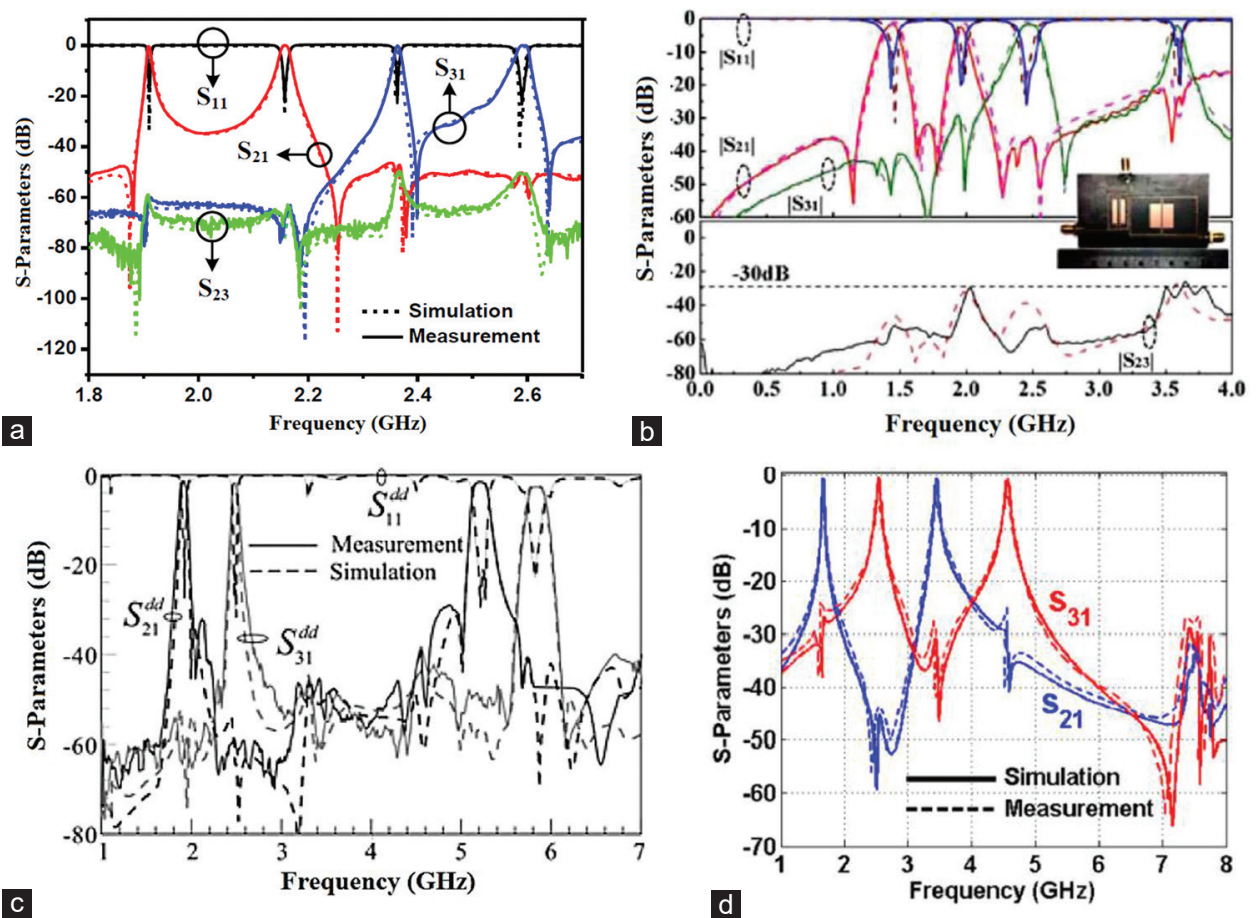

Fig. 4. Simulated and measured frequency response of four-channel diplexers: (a) (Liu, et al., 2017), (b) (Lee, et al., 2016), (c) (Hsu, et al., 2016), and (d) (Noori and Rezaei, 2018).

TABLE IV

Performance and Size Comparison among Different Reported Quad-channel BBD

\begin{tabular}{|c|c|c|c|c|c|c|}
\hline Refs. & ILs (dB) & RLs (dB) & Isolation $(\mathrm{dB})$ & $f_{\mathrm{o} 1}, f_{\mathrm{o} 2}, f_{\mathrm{o} 3}, f_{\mathrm{o} 4}(\mathrm{GHz})$ & Size $\left(\lambda_{\mathrm{g}}^{2}\right)$ & FBWs (\%) \\
\hline (Heng, et al., 2014) & $0.4,0.33,0.35,0.45$ & $19,19,19,20$ & 55 & $2.8,2.81,2.82,2.83$ & 1.17 & $0.2,0.2,0.2,0.2$ \\
\hline (Liu, et al., 2017) & $0.24,0.15,0.18,0.28$ & --- & 50 & $1.9,2.15,2.3,2.5$ & 0.037 & $0.44,0.65,0.45,0.75$ \\
\hline (Lee, et al., 2016) & $1.38,1.6,1.52,1.8$ & --- & 44.8 & $1.92,2.45,5.25,5.81$ & 0.962 & $7.8,6.5,4,3.4$ \\
\hline (Lai and Jeng, 2005) & --- & $10,10,7.75,7.75$ & 10 & $2.52,4.02,5.48,7.13$ & 0.22 & --- \\
\hline (Hsu, et al., 2016) & $2,1.5,2,2.5$ & $>13 \mathrm{~dB}$ & 29 & $0.9,1.5,2.4,3.5$ & 0.042 & $4.3,4.6,3.3,4$ \\
\hline (Noori and Rezaei, 2018) & $0.5,0.38,0.53,0.58$ & $20,21,25,22$ & 30 & $1.67,2.54,3.45,4.57$ & 0.029 & $1.2,1.96,1.15,1.09$ \\
\hline
\end{tabular}

*: Approximated values. BBDS: Bandpass-bandpass diplexers

wave EM simulator has been used to simulate the reported diplexers. As depicted in Fig. 4, the selectivity of the proposed diplexer (Liu, et al., 2017) is poor where the other quadband diplexers (Lee, et al., 2016; Hsu, et al., 2016; Noori and Rezaei, 2018) could improve the frequency selectivity. However, the proposed diplexer (Lee, et al., 2016) has low selectivity at its last channel. On the other hand, any of them could not attenuate the harmonics after the last channel.

The channels with $1 \%$ up to $3 \% \mathrm{FBW}$ are narrowband (Yu and Chang, 1998). However, a narrowband BPF with $0.5 \%$ FBW is proposed (Chen, et al., 2015). As shown in Fig. 4d, the reported diplexer by Noori and Rezaei (2018) has very narrow channels with $1.2 \%, 1.96 \%, 1.15 \%$, and $1.09 \%$ FBWs. The narrow channels of this diplexer give a good resistance against interference. The size and performance of the previously reported quad-channel diplexers are compared. The comparison results are listed in Table IV. The resonance frequency of the quad-channel diplexers at the $1^{\text {st }}, 2^{\text {nd }}, 3^{\text {rd }}$, and $4^{\text {th }}$ channels are presented by $f_{\mathrm{o} 1}, f_{\mathrm{o} 2}, f_{\mathrm{o} 3}$, and $f_{\mathrm{o} 4}$. As written in Table IV, the lowest insertion losses of quad-band diplexers are obtained (Liu, et al., 2017) while the best return losses are obtained (Noori and Rezaei, 2018). Getting high isolation between channels, when we have more number of channels is harder. The highest isolation of quad-channel diplexers is $55 \mathrm{~dB}$, which is obtained (Heng, et al., 2014). Meanwhile, the introduced diplexer (Rezaei, et al., 2019) has the minimum overall size of $0.025 \lambda_{\mathrm{g}}{ }^{2}$ in comparison with the other reported quad-channel diplexers. Among multichannel diplexers, quad-channel diplexers are more designed. However, based on the tri-band unit cell, a six-channel diplexer is proposed in Ghafari and Afsahi, 2019. It operates at $3.4 \mathrm{GHz}, 3.7 \mathrm{GHz}$, $5.6 \mathrm{GHz}, 6 \mathrm{GHz}, 7 \mathrm{GHz}$, and $7.6 \mathrm{GHz}$, which is suitable for wireless and WiMAX applications. Meanwhile, an eightchannel microstrip diplexer with a size of $0.1 \lambda_{\mathrm{g}}{ }^{2}$ is presented in Tu and Hung, 2014. It is designed using coupled closed loops with different widths. The channels of this diplexer are relatively narrow, with isolation between channels better than $29 \mathrm{~dB}$. High selectivity and attenuated harmonics are the advantages of this work. 


\section{LBDS}

The microstrip LBDs are composed of microstrip LPF, BPF, and junction circuit. As mentioned earlier, to achieve a $\mathrm{BBD}$, designing a bandpass resonator was enough. Then, the designed resonator is used to obtain two BPF to use in the diplexer structure.

However, it is necessary to design two bandpass and lowpass resonators to achieve a LBD. The LPF must have high performance with small normalized size. One advantage of a diplexer is its novel structure.
A novel LBD is designed (Rezaei, et al., 2019) for WLAN and WiMAX applications. It is implemented on RT/Duroid 5880 substrate. It includes a novel LPF composed of the patch and thin cells, a BPF, and a small junction circuit. For designing the lowpass section, first, a simple microstrip cell is simulated. Then, it is expanded and simulated step by step so that a very sharp lowpass channel is created. The passband channel of this diplexer is designed based on proposing a basic resonator and its equivalent $L C$ circuit. Then, the even and odd modes of angular resonance

TABLE V

Layout Configuration, Substrate Type, and Theory Method of the LBDs

\begin{tabular}{|c|c|c|c|}
\hline Refs. & Diplexer structure & Substrate & Theory method \\
\hline $\begin{array}{l}\text { (Rezaei, et al., } \\
\text { 2019) }\end{array}$ & & $\begin{array}{c}\text { RT/Duroid } 5880 \\
\varepsilon_{\mathrm{r}}=2.2 \\
h=0.787 \mathrm{~mm}\end{array}$ & $\begin{array}{l}\text { 1. Proposing an } L c \text { model for the basic bandpass resonator } \\
\text { 2. Calculating the angular even/odd modes resonance } \\
\text { frequencies from } L c \text { circuit }\end{array}$ \\
\hline
\end{tabular}

(Rezaei, et al.,

(Deng and Tsai, 2013)

(Heshmati and Roshani, 2018)

(Capstick, 1999)
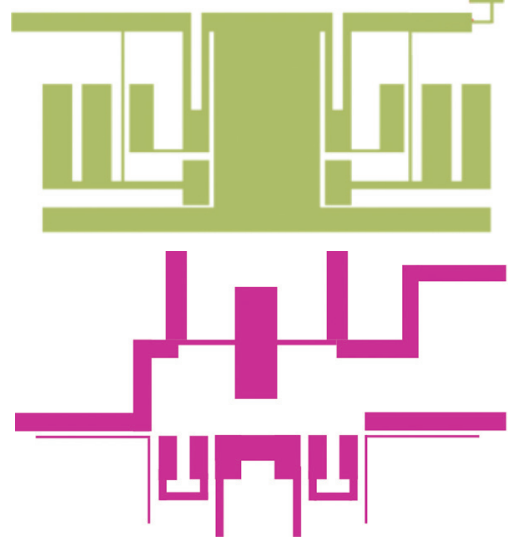

Rogers RO4003C

$\varepsilon_{\mathrm{r}}=3.55$ $h=1.524 \mathrm{~mm}$

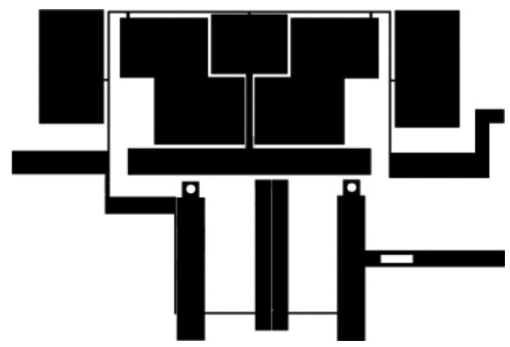

$$
\begin{gathered}
\mathrm{RO} 4003 \mathrm{C} \\
\varepsilon_{\mathrm{r}}=3.38 \\
h=0.813 \mathrm{~mm}
\end{gathered}
$$

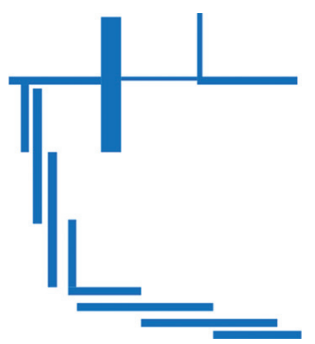

(Hayati, et al., 2019)

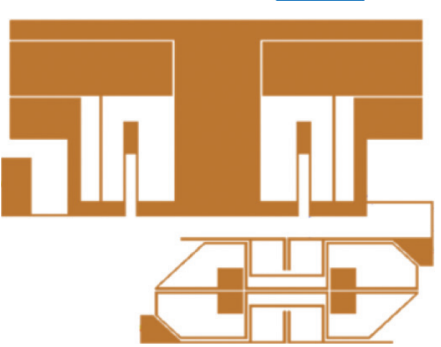

RT/Duroid6002 1. Proposing $L c$ circuits for the LPF and BPF

$\varepsilon_{\mathrm{r}}=2.93 \quad$ 2. Calculating $\mathrm{ABCD}$ matrix of the LPF

$h=30$ mil $\quad 3$. Finding a method to create a lowpass channel

4. Calculating an angular resonance frequency of the BPF

LBDs: Lowpass-bandpass diplexers, LPF: Lowpass filter, BPF: Bandpass filters 
frequencies of the $L C$ model are calculated. Furthermore, a method to attenuate the harmonics is performed similarly to the proposed method (Salehi and Noori, 2015). In this method, all resonance frequencies have been calculated, where the resonance frequency is the main and the others are harmonics. To attenuate these harmonics, the undesirable resonance frequencies and main resonance frequency are set equal. Layout configuration, substrate type, and summarized theory method of the previous reported LBDs are presented in Table V. As depicted in Table V, the use of coupled microstrip cells in the lowpass sections is not essential.

However, the engraved patch cells are utilized (Heshmati and Roshani, 2018; Hayati, et al., 2019). The U-shape cells are coupled to create the bandpass channel (Deng and Tsai, 2013), whereas the bandpass channel (Capstick, 1999 ) is formed by the simple coupled lines. In (Deng and Tsai, 2013), an equivalent circuit model of the proposed LBD is presented. The LPF utilized (Deng and Tsai, 2013) is fifth-order while the electric lengths of the shunt open stub $\left(\theta_{\mathrm{i}}\right)$ and series high-impedance transmission line $\left(\theta_{\mathrm{k}}\right)$ are calculated as follows:

$$
\begin{aligned}
& \theta_{i}=\tan ^{-1}\left(2 \pi f_{c} C_{i} Z_{i}\right) \text { for } i=1,3,5 \\
& \theta_{k}=\sin ^{-1}\left(\frac{2 \pi f_{c} L_{K}}{Z_{K}}\right) \text { for } K=2,4
\end{aligned}
$$

Where $f_{\mathrm{c}}$ is the cutoff frequency of the LPF, $C_{\mathrm{i}}$ and $L_{\mathrm{k}}$ are the required lumped capacitors and inductors, respectively. The parameters $Z_{\mathrm{i}}$ and $Z_{\mathrm{k}}$ are the characteristic impedances. The proposed LBD (Heshmati and Roshani, 2018) is realized based on an optimization method without mathematical analysis. In Capstick, 1999; first, a lowpass prototype is created with the first capacitor $C_{1}$ defined as follows:

$$
C_{1}=\frac{\tan \left(\frac{\pi f_{c}}{2 f_{o}}\right)}{2 \pi f_{c} Z}
$$

Where $f_{\mathrm{c}}, f_{o}$, and $Z$ are the LPF cutoff frequency, the BPF center frequency, and the stub impedance, respectively. Then, the lowpass prototype is transformed into microstrip using the method outlined (Capstick, 1994). After that, a Chebyshev BPF is designed and connected to the LPF. To design the LBD in Hayati, et al., 2019, a perfect mathematical method
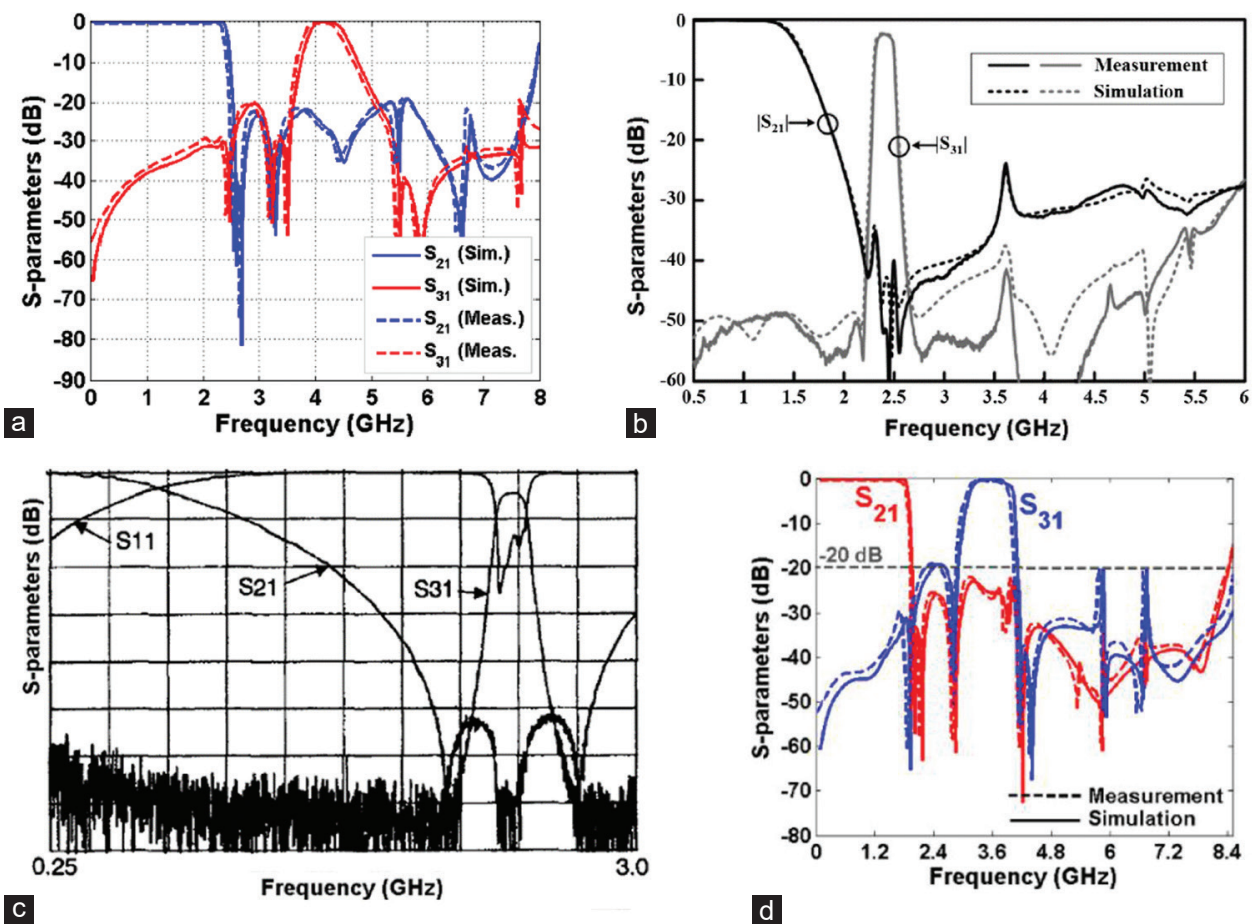

Fig. 5. Simulated and measured frequency responses of four-channel diplexers (a) (Rezaei, et al., 2019), (b) (Deng and Tsai, 2013), (c) (Capstick, 1999), and (d) (Hayati, et al., 2019).

\begin{tabular}{|c|c|c|c|c|c|c|}
\hline Refs. & IL1, IL2 (dB) & RL1, RL2 (dB) & Size $\left(\lambda_{\mathrm{g}}{ }^{2}\right)$ & Isolation $(\mathrm{dB})$ & $f_{\mathrm{c}}, f_{\mathrm{r}}(\mathrm{GHz})$ & $f_{\mathrm{r}} / f_{\mathrm{c}}(\mathrm{GHz})$ \\
\hline (Rezaei, et al., 2019) & $0.15,0.18$ & $18.2,41.4$ & 0.036 & 26 & $2.4,4.2$ & 1.75 \\
\hline (Deng and Tsai, 2013) & $0.25,2.42$ & 15,15 & 0.49 & 35 & $1.5,2.4$ & 1.6 \\
\hline (Rayatzadeh and Moloudian, 2019) & $0.2,0.8$ & --- & --- & 30 & $1,2.4$ & 2.4 \\
\hline (Capstick, 1999) & $1,4.8$ & --- & --- & --- & $0.6,2.4$ & 4 \\
\hline (Hayati, et al., 2019) & $0.12,0.10$ & $19.2,36$ & 0.03 & 20 & $1.88,3.56$ & 1.89 \\
\hline
\end{tabular}

TABLE VI

Performance Comparison BetweEn the LBDs

*: Approximated values. LBD: Lowpass-bandpass diplexers 
is done to design both lowpass and bandpass sections. The approximated equivalent $L C$ models of both lowpass and bandpass resonators are proposed. Then, using the $L C$ circuits, the ABCD matrix for the LPF and the resonance for the bandpass resonator are calculated and analyzed to find the behavior of the device and better tuning the physical dimensions.

The frequency response of the LBDs is depicted in Figs. 5(a)-(d) (Rezaei, et al., 2019; Deng and Tsai, 2013; Capstick, 1999; Hayati, et al., 2019). As shown in Fig. 5, the designed LBDs (Rezaei, et al., 2019; Hayati, et al., 2019) have the advantage of high selectivity, whereas in (Deng and Tsai, 2013; Capstick, 1999) there are poor roll-off at the lowpass channels.

Moreover, the proposed diplexer (Capstick, 1999) could not attenuate the harmonics. Totally, the best selectivity and suppressed harmonics are obtained (Hayati, et al., 2019). The size and performance of the previous reported LBDs are compared in Table VI. In Table VI, IL and RL are the insertion and return losses where the indexes 1 and 2 are related to the lowpass and bandpass channels, respectively. The lowpass channel cutoff frequency and bandpass channel resonance frequency are shown with $f_{\mathrm{c}}$ and $f_{\mathrm{r}}$, respectively. As written in Table VI, the best insertion and return losses are achieved (Hayati, et al., 2019) while it occupies the most compact area $0.03 \lambda_{\mathrm{g}}{ }^{2}$. The highest isolation between channels and the smallest gap between channels are obtained (Deng and Tsai, 2013) by victimizing the overall size so that it has the largest size $\left(0.49 \lambda_{\mathrm{g}}{ }^{2}\right)$.

\section{CONCLUSION}

Several types of microstrip diplexers are studied in this work. The structures and mathematical design methods of these diplexers are reviewed. The reviewed microstrip diplexers are dual-band BBD, LBDs, and multichannel diplexers. Some of the design methods of these works were based on proposing an equivalent $L C$ circuit. Therefore, we presented and explained some proposed $L C$ models of the resonator layouts. The mathematical equations presented in some works were explained too. The layout configurations of the previously reported works with their used substrates were shown and described. The performance of the reviewed diplexers in terms of losses, isolation, selectivity, harmonics, isolation, and the gap between channels is compared. We believe that this review paper may serve a useful guide for researchers who are interested in microstrip diplexers design.

\section{REFERENCES}

Bui, D.H.N., Vuong, T.P., Allard, B., Verdier, J. and Benech, P., 2017. Compact low-loss microstrip diplexer for RF energy harvesting. Electronics Letters, 53(8), pp.552-554.

Bukuru, D., Song, K. and Xue., 2015. Compact wide-stopband planar diplexer based on rectangular dual spiral resonator. Microwave and Optical Technology Letters, 57(1), pp.174-178.

Capstick, M.H., 1994. Simple fast synthesis method for microstrip lowpass filters. Electronics Letters, 30(18), pp.1496-1497.
Capstick, M.H., 1999. Microstrip lowpass-bandpass diplexer topology. Electronics Letters, 35(22), pp.1958-1960.

Chen, C.F., Huang, T.Y., Chou, C.P. and Wu, R.B., 2006. Microstrip diplexers design with common resonator sections for compact size, but high isolation. IEEE Transactions on Microwave Theory and Techniques, 54(5), pp.1945-1952.

Chen, C.F., Zhou, K.W., Chen, R.Y., Wang, Z.C. and He, Y.H., 2019. Design of a microstrip diplexer-integrated filtering power divider. IEEE Access, 7, pp.106514-106520.

Chen, D., Zhu, L., Bu, H. and Cheng, C.H., 2015. A novel planar diplexer using slot line-loaded microstrip ring resonator. IEEE Microwave and Wireless Components Letters, 25(11), pp.706-708.

Chen, J.X., Zhan, Y., Qin, W., Bao, Z.H. and Xue, Q., 2015. Novel narrow-band balanced Bandpass filter using rectangular dielectric resonator. IEEE Microwave and Wireless Components Letters, 25(5), pp.289-291.

Cheng, F., Lin, X., Song, K., Jiang, Y. and Fan, Y., 2013. Compact diplexer with high isolation using the dual-mode substrate integrated waveguide resonator. IEEE Microwave and Wireless Components Letters, 23, pp.459-461.

Chinig, A., 2017. A novel design of microstrip diplexer using meander-line resonators. International Journal of Electronic Engineering and Computer Science, 2(2), pp.5-10.

Chinig, A., Zbitou, J., Errkik, A., Elabdellaoui, L., Tajmouati, A., Tribak, A., and Latrach, M., 2015. A new microstrip diplexer using coupled stepped impedance resonators. International Journal of Electronics and Communication Engineering, 9(1), pp.41-44.

Chinig, A., Zbitou, J., Errkik, A., Tajmouati, A., Abdellaoui, L.E. and Latrach, M., 2015. Microstrip diplexer using stepped impedance resonators. Wireless Personal Communications, 84(4), pp.2537-2548.

Deng, H.W., Zhao, Y.J., Fu, Y., Ding, J. and Zhou, X.J., 2013. Compact and high isolation microstrip diplexer for broadband and WLAN applications. Progress in Electromagnetics Research, 133, pp.555-570.

Deng, P.H. and Tsai, J.T., 2013. Design of microstrip lowpass-bandpass diplexer. IEEE Microwave and Wireless Components Letters, 23(7), pp.332-334.

Feng, W., Gao, X. and Che, W., 2014. Microstrip diplexer for GSM and WLAN bands using common shorted stubs. IET Electronics Letters, 50(20), pp.1486-1488.

Feng, W., Zhang, Y. and Che, W., 2017. Tunable dual-band filter and diplexer based on folded open loop ring resonators. IEEE Transactions on Circuits and Systems, 64(9), pp.1047-1051.

Ghafari, B. and Afsahi, M., 2019. Design of compact multi-channel diplexer using defected microstrip structure. Journal of Communication Engineering, 8(1), pp.138-145.

Guan, X., Yang, F., Liu, H. and Zhu, L., 2014. Compact and high-isolation diplexer using dual-mode stub-loaded resonators. IEEE Microwave and Wireless Components Letters, 24(6), pp.385-387.

Hayati, M., Rezaei, A. and Noori, L., 2019. Design of a high performance lowpass bandpass diplexer using a novel microstrip structure for GSM and WiMAX applications. IET Circuits Devices and Systems, 13(3), pp.361-367.

Heng, Y., Guo, X., Cao, B., Wei, B., Zhang, X., Zhang, G. and Song, X., 2014. A narrowband superconducting quadruplexer with high isolation. IEEE Transactions on Applied Superconductivity, 24(2), pp.21-26.

Heshmati, H. and Roshani, S., 2018. A miniaturized lowpass bandpass diplexer with high isolation. AEÜ International Journal of Electronics and Communications, 87, pp.87-94.

Hsu, K.W., Hung, W.C. and Tu, W.H., 2016. Design of four-channel diplexer using distributed coupling technique. Microwave and Optical Technology Letters, 58(1), pp.166-170.

Huang, F., Wang, J., Zhu, L. and Wu, W., 2016. Compact microstrip balun diplexer using stub-loaded dual-mode resonators. IET Electronic Letters, 52, pp.1994-1996. 
Jun-Mei, Y., Zhou, H.Y. and Cao, L.Z., 2016. Compact diplexer using microstrip half- and quarter wavelength resonators. Electronics Letters, 52(19), pp.16131615.

Lai, M.L. and Jeng, S.K., 2005. A microstrip three-port and four-channel multiplexer for WLAN and UWB coexistence. IEEE Transaction on Microwave Theory and Technique, 53(10), pp.3244-3250.

Lee, C.H., Hsu, C.I.G., Wu, S.X. and Wen, P.H., 2016. Balanced quad-band diplexer with wide common-mode suppression and high differential-mode isolation. IET Microwaves Antennas and Propagation, 10(6), pp.599-603.

Liu, H., Zhu, S., Wen, P., Zhang, X., Sun, L. and Xu, H., 2017. Design of quadchannel high-temperature superconducting diplexer using spiral stub-loaded resonators. IEEE Transactions on Applied Superconductivity, 27(4), pp.75-79.

Lobato-Morales, H., Sun, J.S., Corona-Chavez, A., Itoh, T. and OlveraCervantes, J.L., 2012. UWB and WiLAN microstrip diplexer for differentialmode operation. 2012 IEEE/MTT-S International Microwave Symposium Digest.

Noori, L. and Rezaei, A., 2017. Design of a microstrip diplexer with a novel structure for WiMAX and wireless applications. AEU-International Journal of Electronics and Communications, 77, pp.18-22.

Noori, L. and Rezaei, A., 2017. Design of a microstrip dual-frequency diplexer using microstrip cells analysis and coupled lines components. International Journal of Microwave and Wireless Technologies, 9(7), pp.1467-1471.

Noori, L. and Rezaei, A., 2017. Design of microstrip wide stopband quad-band bandpass filters for multi-service communication systems. AEU-International Journal of Electronics and Communications, 81, pp.136-142.

Noori, L. and Rezaei, A., 2018. Design of a compact narrowband quad-channel diplexer for multi-channel long-range RF communication systems. Analog Integrated Circuits and Signal Processing, 94(1), pp.1-8.

Peng, H. and Chiang, Y., 2015. Microstrip diplexer constructed with new types of dual-mode ring filters. IEEE Microwave and Wireless Components Letters, 25(1), pp.7-9.

Rayatzadeh, S. and Moloudian, G., 2019. Design and fabrication of a miniaturized lowpass-bandpass diplexer with wide tuning range and high isolation, Journal of Electromagnetic Waves and Applications, 33, pp.1874-1889.

Rezaei, A. and Noori, L., 2018. Compact low-loss microstrip diplexer using novel engraved semi-patch cells for GSM and WLAN applications. AEU-International Journal of Electronics and Communications, 87, pp.158-163.

Rezaei, A. and Noori, L., 2018. Miniaturized microstrip diplexer with high performance using a novel structure for wireless L-band applications. Wireless Networks, 26, pp.1-8.

Rezaei, A. and Noori, L., 2018. Novel compact microstrip diplexer for GSM applications. International Journal of Microwave and Wireless Technologies, 10(3), pp.313-317.

Rezaei, A. and Noori, L., 2018. Novel microstrip quadruplexer with wide stopband for WiMAX applications. Microwave and Optical Technology Letters, 60(6), pp.1491-1495.

Rezaei, A., Noori, L. and Jamaluddin, M.H., 2019. Novel microstrip lowpassbandpass diplexer with low loss and compact size for wireless applications. $A E U$ International Journal of Electronics and Communications, 101, pp.152-159.

Rezaei, A., Noori, L. and Mohamadi, H., 2017. Design of a novel compact microstrip diplexer with low insertion loss. Microwave and Optical Technology Letters, 59(7), pp.1672-1676.

Rezaei, A., Noori, L. and Mohammadi, H., 2019. Design of a miniaturized microstrip diplexer using coupled lines and spiral structures for wireless and
WiMAX applications. Analog Integrated Circuits and Signal Processing, 98, pp.409-415.

Rezaei, A., Noori, L. and Mohammadi, H., 2019. Miniaturized quad-channel microstrip diplexer with low insertion loss and wide stopband for multi-service wireless communication systems. Wireless Networks, 25(6), pp.2989-2996.

Rezaei, A., Yahya, S.I., Noori, L. and Jamaluddin, M.H., 2019. Design and fabrication of a novel compact low-loss microstrip diplexer for WCDMA and WiMAX applications. Journal of Microwaves Optoelectronics and Electromagnetic Applications, 18(4), pp.482-491.

Rezaei, A., Yahya, S.I., Noori, L. and Jamaluddin, M.H., 2019. Design of a novel wideband microstrip diplexer using artificial neural network. Analog Integrated Circuits and Signal Processing, 101(1), pp.57-66.

Salehi, M. and Noori, L. 2015. Miniaturized microstrip Bandpass filters using novel stub loaded resonator. Applied Computational Electromagnetics Society, 30(6), pp.692-697.

Salehi, M. and Noori, L., 2014. Novel 2.4 Ghz branch-line coupler using microstrip cells. Microwave and Optical Technology Letters, 56(9), pp.21102113.

Salehi, M.R., Keyvan, S., Abiri, E. and Noori, L., 2016. Compact microstrip diplexer using new design of triangular open loop resonator for $4 \mathrm{G}$ wireless communication systems. AEU International Journal of Electronics and Communications, 70(7), pp.961-969.

Salehi, M.R., Noori, L. and Abiri, E., 2016. Prediction of matching condition for a microstrip subsystem using artificial neural network and adaptive neuro-fuzzy inference system. International Journal of Electronics, 103(11), pp.1882-1893.

Sasipriya, S. and Aparna, C.B., 2018. Compact microstrip diplexer using Bandstop filters for GSM applications. International Journal of Pure and Applied Mathematics, 119(12), pp.1395-1401.

Shen, G. and Che, W., 2020. Compact Ku-band LTCC Bandpass filter using folded dual-composite right and left-handed resonators. Electronics Letters, 56(1), pp.17-19.

Tu, W.H. and Hung, W.C., 2014. Microstrip eight-channel diplexer with wide stopband. IEEE Microwave and Wireless Components Letters, 24(11), pp.742-744.

Wang, X., Wei, B., Zheng, T., Cao, B., Jiang, L. and Chen, J., 2016. Design and implementation of a narrow-band superconducting X-band diplexer with high isolation. Physica C: Superconductivity and its Applications, 531, pp.9-13.

Wu, H.W., Huang, S.H. and Chen, Y.F., 2013. Design of new quad-channel diplexer with compact circuit size. IEEE Microwave and Wireless Components Letters, 23(5), pp.240-242.

Xiao, J.K., Zhu, M., Li, Y., Tian, L. and Ma, J.G., 2015. High selective microstrip bandpass filter and diplexer with mixed electromagnetic coupling. IEEE Microwave and Wireless Components Letters, 25(12), pp.781-783.

Yahya, S.I., Rezaei, A., Noori, L. and Jamaluddin, M.H., 2019. Wide Stopband Microstrip Diplexer Using a Novel Configuration for Frequency Division Duplex and GSM-4G Applications. 2019 International Conference on Engineering, Science, and Industrial Applications, pp.1-15.

Yang, F., Guan, X., Zhu, L. and Liu, H., 2014. Compact microstrip diplexer for 4G Wireless communication. Progress in Electromagnetics Research Symposium Proceedings, 25, pp.599-602.

Yu, C.C. and Chang, K., 1998. Novel compact elliptic-function narrow-band Bandpass filters using microstrip open-loop resonators with coupled and crossing lines. IEEE Transactions on Microwave Theory and Techniques, 46(7), pp.952-958. 